\title{
Estilos parentais e desenvolvimento das funções executivas: estudo com crianças de 3 a 6 anos
}

\author{
Gabriela Lamarca Luxo Martins \\ Camila Barbosa Riccardi León \\ Alessandra Gotuzo Seabra \\ Universidade Presbiteriana Mackenzie, SP, Brasil
}

\begin{abstract}
Resumo
Este estudo analisou a relação entre estilo parental dos responsáveis e funções executivas em pré-escolares, pois pesquisas prévias sugerem que características do ambiente familiar podem influenciar o desenvolvimento dessas funções. Participaram 30 crianças, de 3 a 6 anos, e seus responsáveis. Estes responderam o Inventário de Estilos Parentais e as crianças, Teste de Stroop Semântico, Teste de Atenção por Cancelamento e Teste de Trilhas para pré-escolares. Correlações de magnitude baixa a moderada evidenciaram, de modo geral, que quanto mais apropriado o estilo parental dos pais, melhor o controle de interferência dos filhos. Também observou-se que, quanto maior a inconsistência na punição, maior o tempo de reação da criança na parte incongruente do Teste de Stroop e mais ela é afetada pela incongruência, porém, conforme a parte B do Teste de Trilhas, maior a sua flexibilidade. Portanto observaram-se relações entre estilo parental e funções executivas na amostra avaliada.

Palavras-chave: Relações familiares; Programas educacionais; Funções mentais; Crianças em idade pré-escolar; Neuropsicologia.
\end{abstract}

\section{Parenting styles and development of executive functions: study with 3-6-year-old children}

\begin{abstract}
This study examined the relation between the parenting style of the parents and executive function in preschool children, because previous research suggests that family environment characteristics may influence the development of these functions. Participants were 30 3-6-year-old children and their parents. The parents answered the Parental Styles Inventory and children were assessed by Semantic Stroop Test, Cancellation Attention Test for and Trail Making Test for preschoolers. Low to moderate magnitude correlations showed in general that the more suitable parenting style of the parents, the better interference control of children. It was also observed that the greater the inconsistency in punishment, the greater the child's reaction time in incongruous part of the Stroop Test and the more she/he is affected by the incongruity, however, pursuant to part B of the Trail Making Test, the greater her/ his flexibility. Therefore there were observed relation between parenting style and executive functions of the sample investigated.
\end{abstract}

Keywords: Family relations; Educational programs; Mental functions; Preschool age children; Neuropsychology.

\section{Estilos parentales y desarrollo de funciones ejecutivas: estudio con niños de 3 y 6 años}

\section{Resumen}

Este estudio examinó la relación entre estilos parentales de padres y funciones ejecutivas en niños preescolares, ya que investigaciones anteriores sugieren que las características del entorno familiar pueden influir en el desarrollo de estas funciones. Participaran del estudio 30 niños, entre 3 y 6 años de edad, y sus padres. Los padres respondieron el Inventario de Estilos Parentales y los niños, Test de Stroop Semántico, Test de Atención por Cancelación y Test de Trazados para preescolares. Correlaciones de baja a moderada magnitud mostraron, en general, que cuanto más adecuado el estilo parental de los padres mejor es el control de la interferencia de los niños. También se observó que cuanto mayor es la falta de coherencia en el castigo, mayor es el tiempo de reacción del niño en la parte incongruente del Test de Stroop y más él es afectado por la incongruencia, sin embargo, de conformidad con la parte B del Test de Trazados, mayor es su flexibilidad. Por lo tanto, se observaron relación entre estilo parental y funciones ejecutivas en la muestra investigada.

Palabras clave: Relaciones familiares; Programas educacionales; Funciones mentales; Niños en edad pre-escolar; Neuropsicología. 


\section{Introdução}

As experiências que ocorrem durante os primeiros anos de vida entre pais e filhos são cruciais para o desenvolvimento do cérebro e, mais especificamente, para o desenvolvimento do lobo frontal e suas conexões, caracterizando as bases para, posteriormente, desenvolver as funções executivas (Bernier, Carlson, Deschênes, \& Matte-Gagné, 2012; Bernier, Whipple, \& Carlson, 2010; Oxford \& Lee, 2011). O conjunto das habilidades cognitivas que permitem ao sujeito direcionar comportamentos a metas, avaliar a eficiência e a adequação desses comportamentos, eleger estratégias adequadas e a resolver problemas é denominado de funções executivas (Malloy-Diniz, Sedo, Fuentes, \& Leite, 2008). Tais habilidades estão presentes no controle e na regulação de outros processos comportamentais, os quais abrangem emoção e cognição, e são requisitadas sempre que o sujeito se compromete com determinadas tarefas ou situações novas, sem conhecimento prévio (Diamond, 2013; Lezak, Howieson, Bigler, \& Tranel, 2012; Malloy-Diniz et al., 2008).

Muitas pesquisas atuais abordam temas relacionados às funções executivas (Ardila, 2008; Best \& Miller, 2010; Czermainski, Bosa, \& Salles, 2013; Diamond, 2013; Dias \& Seabra, 2013; Dias \& Seabra, 2015; Dias et al., 2015; Montiel et al., 2014) e outras discutem as configurações familiares e práticas educativas parentais (Leme, Del Prette, \& Coimbra, 2013; Sampaio \& Gomide, 2007; Oxford \& Lee, 2011), porém poucas se referem aos dois temas concomitantemente (Bernier, Whipple \& Carlson, 2010; Bernier et al., 2012; Bibok, Carpendale, \& Müller, 2009; Bindman, Hindman, Bowles, \& Morrison, 2013; Rhodes et al., 2011). Assim sendo, ao considerar os vários fatores ambientais que se relacionam com o desenvolvimento das funções executivas, as evidências sugerem que a orientação dos pais, ou seja, a conduta utilizada pelos progenitores para direcionar o processo de educação de seus filhos é considerada uma das experiências primárias mais relevantes para a constituição dessas funções durante os primeiros anos da infância (Bernier, Whipple, \& Carlson, 2010; Bernier et al., 2012). Os progenitores, por meio de características específicas, auxiliam a criança a atingir um nível de funções executivas além do que ela poderia conseguir sozinha (Bindman et al., 2013).

Pesquisas têm sido conduzidas analisando diferentes características dos pais e da sua relação com as crianças, tais como a relação de apego, a sensibilidade materna, os estilos parentais, histórico acadêmico materno e condição social (Bernier, Whipple, \&
Carlson, 2010; Carvalho et al., 2012; Osório, 2011). Por exemplo, Bernier et al. (2012) estudaram a relação de apego, conceituada como o resultado de um comportamento de cuidado parental que pode ser observado a partir de comportamentos da própria criança, tais como lidar com a frustração no momento de realizar uma tarefa difícil ou ao adiar a gratificação com base na confiança no cuidador, o qual pode explicar que a atividade desejada será realizada em breve. A qualidade desse vínculo afetivo fornece um contexto seguro e ordenado de relacionamento em que as crianças podem, gradualmente, aprender a usar o pensamento autorregulado e desenvolver as ações que definem as funções executivas. Consequentemente, segundos autores, a qualidade das interações com os pais está relacionada ao desenvolvimento das funções executivas das crianças.

Osório (2011), em sua tese, observou que, já aos 10 meses de vida, as estratégias maternas em relação à atenção compartilhada são um preditor significativo para o seguimento de atenção compartilhada pelo bebê. Tal afirmação aponta a relevância da figura materna desde os primeiros meses de vida. Também em relação ao processo de interação com os progenitores, Carvalho et al. (2012) abordaram o conceito de scaffolding, o qual, traduzido para a língua portuguesa, significa 'andaimes' e pode ser compreendido como o suporte temporário que os progenitores, educadores ou adultos, ou seja, os tutores na nomenclatura dos autores, fornecem à criança em determinada tarefa, até que esta consiga prosseguir sozinha com destreza.

Outro aspecto destacado como relevante ao desenvolvimento infantil são os estilos parentais. Darling e Steinberg (1993) realizaram uma revisão histórica do conceito de estilo parental e propuseram a compreensão desse conceito como o contexto em que os pais influenciam os seus filhos por meio de suas práticas, levando em consideração suas crenças e valores, ultrapassando a combinação entre exigência e responsividade. No Brasil, Sampaio e Gomide (2007) definiram estilo parental como o conjunto de práticas educativas utilizadas pelos pais na interação com os filhos. Estas autoras selecionaram, em seu modelo teórico, sete práticas educativas que compõem o estilo parental, sendo cinco relacionadas ao desenvolvimento de comportamentos antissociais e duas favoráveis ao desenvolvimento de comportamentos pró-sociais. O presente artigo focalizará esse aspecto do comportamento dos pais e, portanto, as sete práticas educativas encontram-se descritas mais detalhadamente a seguir.

As práticas favoráveis ao desenvolvimento de comportamentos pró-sociais incluem a monitoria 
positiva e o comportamento moral. A primeira consiste no conjunto de práticas parentais que envolvem, por exemplo, atenção e conhecimento dos pais acerca de onde o seu filho se encontra e das atividades desenvolvidas por ele. A segunda refere-se a uma prática educativa pela qual os pais transmitem valores, como honestidade, generosidade e senso de justiça aos filhos, auxiliando-os na discriminação do certo e do errado por meio de modelos positivos, dentro de uma relação de afeto.

As práticas educativas relacionadas ao desenvolvimento de comportamentos antissociais são de cinco tipos. Primeiramente, a negligência, a qual ocorre quando os pais não estão atentos às necessidades de seus filhos, ausentam-se das responsabilidades, omitem-se de auxiliar seus filhos, ou simplesmente quando interagem sem afeto, sem amor. A segunda é a punição inconsistente, a qual consiste em os pais punirem ou reforçarem os comportamentos de seus filhos de acordo com o próprio bom ou mau humor, de forma não contingente ao comportamento da criança. A terceira prática educativa é nomeada de monitoria negativa e está relacionada ao excesso de fiscalização dos pais sobre a vida dos filhos e à grande quantidade de instruções repetitivas. A disciplina relaxada é o quarto tipo e consiste no não cumprimento das regras estabelecidas pelos próprios pais. E a última é o abuso físico, a qual contempla o ato dos pais machucarem ou causarem dor a seus filhos com a justificativa de que estão educando.

Repetidas experiências de regulação de sucesso por parte dos progenitores, em determinadas situações emocionalmente desgastantes, levam as crianças a internalizar as competências adquiridas e gradualmente integrá-las em seu próprio repertório de autorregulação (Bernier et al., 2012; Rhoades, Greenberg, Lanza, \& Blair, 2011). Desta forma, os processos regulatórios tendem a ser, primeiramente, praticados no âmbito das relações familiares; as estratégias aprendidas então tendem a se generalizar e a ser utilizadas fora da relação didática, tal como durante tarefas que exijam autorregulação independente, a qual pode ser considerada uma característica definidora das tarefas de funções executivas (Bernier et al., 2012). Pode-se supor, por meio do estudo de Bernier et al. (2012), que os fatores mais proximais, como, por exemplo, as funções executivas dos próprios pais, possam afetar o controle das crianças referente ao próprio impulso, o que pode ocorrer por meio de imitação e de aprendizagem por observação.

No estudo de Rhoades, Greenberg, Lanza e Blair (2011) destacou-se que poucas pesquisas inserem o desenvolvimento das funções executivas em um dos contextos mais importantes para oportunizar o mesmo, isto é, no ambiente doméstico. Tal estudo enfocou os indicadores demográficos e o desenvolvimento das funções executivas no ambiente familiar e, no mesmo, identificou perfis familiares de risco, incluindo as práticas parentais e a estrutura familiar. Os resultados sugeriram que combinações específicas de risco são mais associadas com baixos resultados de funções executivas e que essas combinações podem variar de acordo com a raça, estado civil e condição socioeconômica. Observou-se, por exemplo, que a qualidade da parentalidade pode exercer influência no desenvolvimento das crianças de duas maneiras. Em primeiro lugar, por meio de interações sensíveis contingentes com os progenitores, afinal, por meio destas, a criança pode aumentar a sua motivação para aprender a controlar e interagir com o mundo externo, o que pode contribuir para a prática das habilidades relacionadas às funções executivas. Em um segundo momento, por meio da segurança derivada de interações positivas, neste caso as crianças são mais propensas a explorar o seu ambiente, aumentando a sua interação com materiais que a estimulem cognitivamente (Rhoades et al., 2011).

A pesquisa de Sarsour et al. (2011) teve como objetivo investigar as contribuições independentes e interativas do status socioeconômico e status monoparentais das famílias a nível do funcionamento executivo das crianças. Os autores comentam sobre a pesquisa de Ardila et al. (2005), os quais descobriram que os anos da escolaridade dos pais foram associados com o desempenho das funções executivas de crianças e foi encontrada relação mais predominante para fluência verbal semântica. Outro estudo complementa que a habilidade da linguagem da criança pode mediar a relação entre o status socioeconômico da família e a função executiva da criança (Noble et al., 2007). Por fim, Sarsour et al. (2011) comentam que o status socioeconômico pode afetar a medida em que os pais usam os recursos da família para enriquecer as experiências de desenvolvimento com passatempos, recreação, museus, bibliotecas, viagens, etc. Além disso, o status socioeconômico da família pode afetar dimensões parentais, incluindo a capacidade de resposta emocional e verbal dos pais, oferecendo reforço para o comportamento desejado e fornecendo andaimes para incentivar o desenvolvimento das habilidades executivas.

Tendo em vista a relevância das práticas parentais para o desenvolvimento infantil e, especificamente, para o desenvolvimento das funções executivas, e diante da escassez de estudos nessa área principalmente no âmbito nacional, este estudo objetivou verificar 
as relações entre as práticas educativas dos pais (em termos de monitoria positiva, comportamento moral, punição inconsistente, negligência, disciplina relaxada, monitoria negativa e abuso físico) e as funções executivas em crianças pré-escolares.

\section{Método}

\section{Participantes}

Participaram deste estudo crianças de 3 a 6 anos de idade, que cursavam os níveis de Maternal II, Nível I e Nível II de uma escola particular de Educação Infantil localizada na cidade de São Paulo. Os responsáveis por todas as 42 crianças matriculadas nessas séries foram convidados a participar. Desses, 10 não autorizaram a participação de seus filhos na pesquisa e, ao longo do ano letivo, 2 crianças saíram da escola, chegando à amostra final de 30 crianças e seus respectivos responsáveis.

Dessa forma, a amostra final foi constituída por 60 sujeitos, sendo 30 crianças entre 03 e 06 anos de idade, com média de 4,22 anos, de ambos os sexos, e seus respectivos pais. A amostra foi por conveniência e o número de participantes selecionados foi para fins de exploração dos dados, não sendo derivado de cálculo amostral. A Tabela 1 indica a caracterização da amostra de crianças por idade, nível escolar, gênero e composição familiar e a caracterização da amostra de responsáveis pelas crianças que contempla: grau de parentesco dos participantes, estado civil e nível educacional de mãe e pai.

\section{Instrumentos}

\section{Questionário de identificação}

Por meio deste, desejou-se obter informações relevantes que não foram abordadas nos instrumentos utilizados, entre elas, dados pessoais (nome da mãe, do pai e data de nascimento da criança), escolaridade dos pais e organização familiar (estado civil dos pais, quantidade de pessoas que habitam a casa, quem convive no ambiente familiar e número de irmãos).

\section{Inventário de Estilos Parentais (IEP)}

(Gomide, 2011b)

Avalia o estilo parental, ou seja, as estratégias e técnicas utilizadas pelos pais para educar os filhos. O inventário é composto por 42 questões que correspondem às sete práticas educativas utilizadas pelos pais na interação com os filhos. Para cada prática educativa, foram elaboradas seis questões distribuídas espaçadamente ao longo do inventário. São duas práticas educativas positivas: (A) monitoria positiva e (B) comportamento moral, e cinco práticas educativas negativas: (C) punição inconsistente, (D) negligência, (E) disciplina relaxada, $(F)$ monitoria negativa e $(\mathrm{G})$ abuso físico (Gomide, 2011b).

TABELA 1

Caracterização da amostra de crianças por idade, gênero e composição familiar e caracterização dos responsáveis pelas crianças, que participaram do estudo, em relação ao grau de parentesco, estado civil e nível educacional de mãe e pai.

\begin{tabular}{|c|c|c|c|c|c|c|c|c|c|c|}
\hline \multicolumn{11}{|c|}{ Caracterização da amostra de crianças } \\
\hline \multirow[b]{2}{*}{ Série } & \multirow{2}{*}{$\begin{array}{l}\text { Idade média } \\
\text { (mín-máx) }\end{array}$} & \multirow[b]{2}{*}{$D P$} & \multirow[b]{2}{*}{ Mediana } & \multicolumn{2}{|c|}{ Gênero } & \multicolumn{5}{|c|}{ Composição familiar } \\
\hline & & & & Masc. & Fem. & Mãe/Pai & $\begin{array}{c}\text { Mãe/Pail } \\
\text { Outros }\end{array}$ & \multicolumn{2}{|c|}{ Mãe elou Pai } & Sem pais \\
\hline Mat. II & $\begin{array}{c}3,42 \\
(3,04-4,11)\end{array}$ & 0,49 & 3,08 & 5 & 9 & 6 & 7 & \multicolumn{2}{|c|}{1} & 0 \\
\hline Nível I & $\begin{array}{c}4,30 \\
(4,04-5,11)\end{array}$ & 0,46 & 4,06 & 5 & 3 & 4 & 4 & \multicolumn{2}{|c|}{0} & 0 \\
\hline Nível II & $\begin{array}{c}5,56 \\
(5,03-6,10)\end{array}$ & 0,53 & 5,54 & 5 & 3 & 4 & 2 & \multicolumn{2}{|c|}{1} & 1 \\
\hline Total & $\begin{array}{c}4,22 \\
(3,04-6,10)\end{array}$ & 1,02 & 4,06 & 15 & 15 & 14 & 13 & \multicolumn{2}{|c|}{2} & 1 \\
\hline \multicolumn{10}{|c|}{ Caracterização da amostra de responsáveis } & \\
\hline \multirow[b]{2}{*}{ Série } & \multicolumn{3}{|c|}{ Grau de parentesco } & \multicolumn{2}{|c|}{ Estado civil } & \multicolumn{4}{|c|}{ Nivel educacional de mãe e pai $(n=60)$} & \\
\hline & $\begin{array}{c}\text { Responsável } \\
\text { legal }\end{array}$ & Mãe & $P a i$ & Casado & Div. & $\begin{array}{l}\text { Ensino } \\
\text { médio }\end{array}$ & $\begin{array}{c}\text { Graduação } \\
\text { incompletal } \\
\text { completa }\end{array}$ & Esp. & Mestre & \\
\hline Mat. II & 0 & 13 & 1 & 13 & 1 & 2 & 18 & 8 & 0 & \\
\hline Nível I & 0 & 6 & 2 & 8 & 0 & 1 & 9 & 5 & 1 & \\
\hline Nível II & 1 & 7 & 0 & 6 & 2 & 2 & 9 & 3 & 2 & \\
\hline Total & 1 & 26 & 3 & 27 & 3 & 5 & 36 & 16 & 3 & \\
\hline
\end{tabular}


O IEP possui duas formas de aplicação. Há um inventário de estilos parentais denominado "práticas educativas paternas e maternas" para que os pais respondam sobre as práticas educativas adotadas em relação ao filho e, existem dois inventários, através dos quais os filhos, acima de oito anos, podem responder sobre as práticas educativas utilizadas por seus pais. Os pais poderão responder sobre os filhos acima de cinco anos desde que façam as devidas adaptações às situações propostas pelo inventário (Gomide, 2011b). Neste estudo foi utilizado o formato em que os pais respondem sobre o próprio filho e foram realizadas adaptações em 12 itens, com autorização prévia da autora, apenas para uma melhor compreensão do conceito por parte dos pais, sem alteração substancial de conteúdo. As modificações foram executadas para uma melhor compreensão, por parte dos pais, sobre a realidade de seus filhos, afinal tratava-se de um vocabulário para uma faixa etária superior, como, por exemplo, se os pais explicavam ao filho que era melhor tirar uma nota baixa do que colar em uma prova; ou se, quando o filho saía com alguém, os pais ligavam muitas vezes, etc. Foram adaptados os itens de número $1,9,13,18,20,23,26,29,33,34,37$ e 38 da versão original do instrumento.

O cálculo do índice de estilo parental é realizado a partir das práticas positivas $(\mathrm{A}+\mathrm{B})$ e as práticas negativas $(\mathrm{C}+\mathrm{D}+\mathrm{E}+\mathrm{F}+\mathrm{G})$ e, em seguida, subtrai-se a soma das práticas negativas das positivas. Desta forma, quando o índice de estilo parental é negativo, indica prevalência de práticas parentais negativas que neutralizam ou se sobrepõem às práticas parentais positivas. Quando o índice de estilo parental é positivo, aponta uma forte presença de práticas parentais positivas que se sobrepõem às práticas negativas. Evidência de validade podem ser verificadas em Gomide (2011b).

\section{Teste de Stroop Semântico (TSS)}

O Teste de Stroop Semântico, utilizado nesse estudo, é uma versão adaptada por Trevisan (2010) com base nas versões de Berwid et al. (2005), Brocki e Bohlin (2006), Gerstadt, Hong e Diamond (1994), assim como o Stroop clássico (Stroop, 1935). O instrumento é computadorizado e o objetivo consiste em avaliar a atenção seletiva e o controle inibitório de crianças. Pode ser utilizado com crianças alfabetizadas ou não, pois não faz o uso de material escrito. O TSS é indicado para crianças de 3 a 7 anos de idade.

A versão original é composta por palavras, porém esta é constituída por dois pares de figuras: sol e lua, menino e menina. O teste é dividido em duas partes, primeiramente, a criança deve nomear as figuras e, posteriormente, a mesma deve dizer o oposto. Após nomear, no momento em que visualizar o dia ela deverá dizer noite e quando visualizar o menino deverá dizer menina, assim por diante (Trevisan, 2010).

Ao total é composto por 32 imagens, divididas em 16 para a primeira etapa e 16 para a segunda etapa e vale lembrar que cada etapa contém uma progressão de dificuldade. Na primeira etapa as imagens aparecem com um intervalo de 1200 milésimos de segundo e na segunda as imagens aparecem em um intervalo de 800 milésimos de segundo (Martoni, 2012). Evidência de validade podem ser verificadas em Trevisan (2010).

\section{Teste de Atenção por Cancelamento (TAC)}

Avalia a atenção seletiva, atenção alternada, planejamento e o controle inibitório, o mesmo é composto por três fases, com dificuldade progressiva, nas quais, inicialmente, é apresentada para a criança um estímulo alvo e lhe é solicitada que assinale o mesmo em meio a diversos outros em um espaço delimitado (Trevisan, 2010). É indicado para crianças de 4 a 14 anos de idade e em jovens adultos (20 a 32 anos).

Os acertos são contabilizados quando a criança assinala o estímulo alvo de forma correta e os erros quando ela omite ou assinala algum outro sem ser o desejado. Na primeira fase pode-se acertar de 0 a 60 estímulos, sendo 15 estímulos corretos em cada quadrante. Na segunda fase pode-se acertar de 0 a 12 estímulos, entre eles, 3 estímulos corretos em cada quadrante. Na última fase pode-se acertar de 0 a 60 estímulos, contendo 15 estímulos corretos em cada quadrante. $\mathrm{O}$ instrumento total contém de 0 a 132 pontos corretos (Trevisan, 2010).

$\mathrm{Na}$ primeira fase, no topo da folha, encontra-se o estímulo alvo e abaixo são disponibilizadas 300 imagens de: círculo, quadrado, triângulo, cruz, estrela e traço. Todas as imagens estão organizadas de uma forma aleatória e a criança deve assinalar os estímulos idênticos ao alvo. $\mathrm{Na}$ segunda fase, no lugar do estímulo alvo é solicitado que a criança encontre duas imagens que precisam estar localizadas uma ao lado da outra na mesma linha e em mesma ordem conforme o alvo. Na terceira e última fase, o estímulo alvo se modifica conforme cada linha, sendo necessário que a criança consiga manter a atenção alternada adequada para realizar de forma correta o que é requisitado. Cada criança possui um minuto para realizar cada etapa (Montiel \& Seabra, 2012). Parâmetros psicométricos do TAC são disponibilizados por Godoy (2012); conforme a autora, há evidências de validade para o teste quando aplicado a crianças a partir de 4 anos de idade, alunos do penúltimo ano da Educação Infantil. 
Dados normativos são disponibilizados em Dias, Trevisan, Pereira, Gonzales e Seabra (2012).

\section{Teste de Trilhas para pré-escolares (TTPE)}

Neste estudo foi utilizada a versão do Teste de Trilhas adaptada para pré-escolares de Trevisan (2010), a partir das partes A e B do Teste de Partington e Leiter (1949). Trevisan (2010) fez esta adaptação pautada nas versões de Espy (1997), Espy, Kaufmann e Glisky (2001), Espy e Cwik (2004) e Baron (2004). A versão atual está validada e, como é composta somente por imagens, é indicada para crianças de 4 a 6 anos de idade.

Primeiramente, na Parte A, é apresentada uma folha para a criança com a imagem de uma família composta por cinco cachorros, representando: pai, mãe e três filhotes. A tarefa da criança é fazer a ligação entre os cachorros em ordem de tamanho crescente. Posteriormente, na Parte B, são apresentadas dez figuras, contemplando cinco cachorros e cinco ossos, cada um de um tamanho, porém de forma que dê para agrupar duplas em relação aos tamanhos apresentados. A criança deve fazer um traçado com o objetivo de unir o cachorro e o osso do tamanho correspondente e assim por diante (Trevisan \& Seabra, 2012).

Parâmetros psicométricos do Teste de Trilhas para pré-escolares são disponibilizados por Trevisan e Pereira (2012), revelando evidências de validade para o teste quando aplicado a crianças a partir de 4 anos de idade, cursando o penúltimo ano da Educação Infantil. Dados normativos são disponibilizados em Trevisan, Hipólito, Parise, Reppold e Seabra (2012).

\section{Procedimento}

Após aprovação da pesquisa pelo Comitê de ética em Pesquisa, os responsáveis pela instituição e, posteriormente, os responsáveis pelas crianças foram contatados para assinar ao Termo de Consentimento Livre e Esclarecido.

Em seguida, foi agendado um horário para entrevista individual com a figura materna e/ou paterna e/ou responsável legal de cada família na própria escola, em uma sala destinada para tal atividade e em horários previamente autorizados pela mesma. $\mathrm{O}$ encontro teve a duração de, aproximadamente, 30 minutos. Nele foi solicitado que o responsável preenchesse o questionário de identificação e o Inventário de Estilo Parental (IEP).

Em relação à avaliação das crianças, no Maternal foi aplicado apenas o Teste de Stroop Semântico, visto que os demais testes não apresentam, como já descrito nos Instrumentos, parâmetros psicométricos aceitáveis para a faixa etária de 3 anos, com propriedades aceitáveis para crianças a partir de 4 anos alunas do penúltimo ano da Educação Infantil. Nos Níveis I e II foram aplicados os três instrumentos de funções executivas. Levando em consideração a idade da amostra e conforme solicitado pela instituição, foi aplicado um teste por encontro, totalizando três encontros individuais para Níveis I e II e um encontro para o Maternal, com a duração de, aproximadamente, 20 minutos cada um, realizados nos intervalos de aulas das respectivas professoras. Vale salientar que a aplicação de um próximo instrumento só foi realizada após a conclusão do anterior em todas as crianças participantes.

A retirada de alunos da sala de aula para os encontros de avaliação foi sempre realizada com autorização prévia da educadora, assim como com a aceitação do próprio aluno. Os testes foram aplicados em todas as crianças sempre na mesma ordem, para evitar que possíveis efeitos de aprendizagem escolar promovessem diferenças nos escores nos testes entre os grupos de crianças. Para evitar efeito de fadiga, foram conduzidas três sessões com as crianças. No primeiro encontro foi aplicado o Teste de Stroop Semântico; no segundo, o Teste de Trilhas para préescolares e, no terceiro encontro, o Teste de Atenção por Cancelamento.

\section{Resultados}

Para proceder às análises objetivadas, inicialmente foi calculada a normalidade da distribuição. Conforme os testes de normalidade de Kolmogorov-Smirnov e Shapiro-Wilk, a normalidade não foi confirmada. Assim sendo, foram conduzidas estatísticas não paramétricas. Para verificar a relação entre os escores do Inventário de Estilos Parentais e dos testes que avaliaram as funções executivas das crianças, foi conduzida correlação nãoparamétrica de Spearman.

No que se refere ao IEP e ao Teste de Stroop Semântico (Tabela 2) algumas correlações podem ser elencadas. O percentual do IEP revelou correlação negativa significativa, de magnitude moderada, com a interferência no tempo de reação do TSS. A prática educativa negativa, denominada punição inconsistente, apresentou correlação positiva significativa, de magnitude baixa, com a média do tempo de reação na parte 2 do TSS; a mesma prática educativa expressou correlação positiva significativa, de magnitude moderada, com a interferência no tempo de reação do TSS. Por fim, a prática educativa negativa, denominada abuso físico, revelou correlação positiva significativa, de magnitude moderada, com a média do tempo de reação na parte 2 e com a interferência no tempo de reação, ambos do TSS. 
Em relação ao IEP e ao Teste de Atenção por Cancelamento (Tabela 3), houve correlação positiva significativa, de magnitude moderada, entre o percentual total do IEP e o total de acertos na parte 2 do TAC.
Quanto ao IEP e ao Teste de Trilhas para préescolares (Tabela 4) a prática educativa negativa, nomeada de punição inconsistente, revelou correlação positiva significativa, de magnitude moderada, com as conexões da parte B do TTPE.

TABELA 2

Análise de correlação não-paramétrica de Spearman entre o desempenho no Inventário de Estilos Parentais (IEP) e no Teste de Stroop Semântico $(\mathrm{N}=30)$.

\begin{tabular}{|c|c|c|c|c|c|c|c|}
\hline & & $\begin{array}{l}\text { Stroop } \\
\text { Parte } 1 \\
\text { Acertos }\end{array}$ & $\begin{array}{c}\text { Stroop } \\
\text { Parte } 1 \\
\text { Tempo de Reação }\end{array}$ & $\begin{array}{l}\text { Stroop } \\
\text { Parte } 2 \\
\text { Acertos }\end{array}$ & $\begin{array}{c}\text { Stroop } \\
\text { Parte } 2 \\
\text { Tempo de Reação }\end{array}$ & $\begin{array}{c}\text { Interferência } \\
\text { Escore }\end{array}$ & $\begin{array}{c}\text { Interferência } \\
\text { Tempo de Reação }\end{array}$ \\
\hline \multirow{2}{*}{ Percentual } & rho & $-0,144$ & 0,061 & 0,027 & $-0,347$ & 0,112 & $-0,435 *$ \\
\hline & $\mathrm{p}$ & 0,457 & 0,754 & 0,891 & 0,076 & 0,562 & 0,023 \\
\hline \multirow{2}{*}{$\begin{array}{l}\text { Monitoria } \\
\text { Positiva }\end{array}$} & rho & $-0,207$ & 0,171 & 0,011 & $-0,048$ & 0,059 & $-0,148$ \\
\hline & $\mathrm{p}$ & 0,282 & 0,374 & 0,953 & 0,812 & 0,76 & 0,461 \\
\hline \multirow{2}{*}{$\begin{array}{l}\text { Compto. } \\
\text { Moral }\end{array}$} & rho & $-0,05$ & $-0,044$ & 0,235 & 0,216 & 0,271 & 0,18 \\
\hline & $\mathrm{p}$ & 0,795 & 0,82 & 0,22 & 0,28 & 0,154 & 0,37 \\
\hline \multirow{2}{*}{$\begin{array}{l}\text { Punição } \\
\text { Inconsistente }\end{array}$} & rho & 0,141 & $-0,15$ & 0,113 & $0,393 *$ & 0,053 & $0,518 * *$ \\
\hline & $\mathrm{p}$ & 0,467 & 0,437 & 0,558 & 0,042 & 0,785 & 0,006 \\
\hline \multirow{2}{*}{ Negligência } & rho & 0,193 & $-0,134$ & 0,15 & 0,113 & 0,077 & 0,152 \\
\hline & $\mathrm{p}$ & 0,316 & 0,489 & 0,437 & 0,576 & 0,693 & 0,448 \\
\hline \multirow{2}{*}{$\begin{array}{l}\text { Disciplina } \\
\text { Relaxada }\end{array}$} & rho & 0,091 & 0,001 & $-0,165$ & 0,21 & $-0,22$ & 0,253 \\
\hline & $\mathrm{p}$ & 0,639 & 0,996 & 0,393 & 0,294 & 0,252 & 0,202 \\
\hline \multirow{2}{*}{$\begin{array}{l}\text { Monitoria } \\
\text { Negativa }\end{array}$} & rho & $-0,135$ & $-0,185$ & 0,095 & $-0,019$ & 0,062 & $-0,003$ \\
\hline & $\mathrm{p}$ & 0,486 & 0,336 & 0,625 & 0,924 & 0,749 & 0,986 \\
\hline \multirow{2}{*}{ Abuso Físico } & rho & 0,221 & 0,206 & 0,206 & $0,438 *$ & 0,174 & $0,428 *$ \\
\hline & $\mathrm{p}$ & 0,249 & 0,284 & 0,285 & 0,022 & 0,367 & 0,026 \\
\hline
\end{tabular}

$* \mathrm{p} \leq 0,05 ; * * \mathrm{p} \leq 0,01$

TABELA 3

Análise de correlação não-paramétrica de Spearman entre o desempenho no Inventário de Estilos Parentais (IEP) e no Teste de Atenção por Cancelamento $(\mathrm{N}=16)$.

\begin{tabular}{|c|c|c|c|c|c|}
\hline & & $\begin{array}{c}\text { TAC } \\
\text { Parte } 1 \\
\text { Acertos }\end{array}$ & $\begin{array}{c}\text { TAC } \\
\text { Parte } 2 \\
\text { Acertos }\end{array}$ & $\begin{array}{c}\text { TAC } \\
\text { Parte } 3 \\
\text { Acertos }\end{array}$ & $\begin{array}{c}\text { TAC } \\
\text { Total } \\
\text { Acertos }\end{array}$ \\
\hline \multirow{2}{*}{ Percentual } & rho & 0,233 & $0,515^{*}$ & 0,232 & 0,204 \\
\hline & $\mathrm{p}$ & 0,384 & 0,041 & 0,388 & 0,449 \\
\hline \multirow{2}{*}{ Monitoria Positiva } & rho & 0,049 & 0,261 & 0,119 & 0,102 \\
\hline & $\mathrm{p}$ & 0,856 & 0,328 & 0,662 & 0,708 \\
\hline \multirow{2}{*}{ Comportamento Moral } & rho & $-0,019$ & 0,251 & 0,131 & 0,05 \\
\hline & $\mathrm{p}$ & 0,944 & 0,348 & 0,628 & 0,854 \\
\hline \multirow{2}{*}{ Punição Inconsistente } & rho & 0,109 & $-0,249$ & 0,062 & 0,181 \\
\hline & $\mathrm{p}$ & 0,688 & 0,352 & 0,818 & 0,503 \\
\hline \multirow{2}{*}{ Negligência } & rho & 0,044 & 0,057 & 0,081 & 0,095 \\
\hline & $\mathrm{p}$ & 0,871 & 0,835 & 0,767 & 0,725 \\
\hline \multirow{2}{*}{ Disciplina Relaxada } & rho & $-0,389$ & $-0,451$ & $-0,288$ & $-0,357$ \\
\hline & $\mathrm{p}$ & 0,137 & 0,08 & 0,279 & 0,175 \\
\hline \multirow{2}{*}{ Monitoria Negativa } & rho & $-0,057$ & $-0,125$ & 0,347 & 0,223 \\
\hline & $\mathrm{p}$ & 0,835 & 0,646 & 0,187 & 0,406 \\
\hline \multirow{2}{*}{ Abuso Físico } & rho & 0,226 & $-0,077$ & 0,016 & 0,148 \\
\hline & $\mathrm{p}$ & 0,4 & 0,777 & 0,952 & 0,586 \\
\hline
\end{tabular}

$* \mathrm{p} \leq 0,05 ; * * \mathrm{p} \leq 0,01$. 
TABELA 4

Análise de correlação não-paramétrica de Spearman entre o desempenho no Inventário de Estilos Parentais (IEP) e no Teste de Trilhas para pré-escolares $(\mathrm{N}=16)$.

\begin{tabular}{|c|c|c|c|c|c|}
\hline & & $\begin{array}{l}\text { Teste de Trilhas } \\
\text { Parte A } \\
\text { Conexões }\end{array}$ & $\begin{array}{l}\text { Teste de Trilhas } \\
\text { Parte A } \\
\text { Sequências }\end{array}$ & $\begin{array}{c}\text { Teste de Trilhas } \\
\text { Parte B } \\
\text { Conexões }\end{array}$ & $\begin{array}{l}\text { Teste de Trilhas } \\
\text { Parte B } \\
\text { Sequências }\end{array}$ \\
\hline \multirow{2}{*}{ Percentual } & rho & 0,131 & 0,131 & $-0,322$ & $-0,193$ \\
\hline & $\mathrm{p}$ & 0,63 & 0,63 & 0,224 & 0,473 \\
\hline \multirow{2}{*}{ Monitoria Positiva } & rho & $-0,192$ & $-0,192$ & $-0,276$ & $-0,299$ \\
\hline & $\mathrm{p}$ & 0,475 & 0,475 & 0,3 & 0,261 \\
\hline \multirow{2}{*}{ Compto. Moral } & rho & 0,051 & 0,051 & 0,14 & 0,059 \\
\hline & $\mathrm{p}$ & 0,852 & 0,852 & 0,606 & 0,829 \\
\hline \multirow{2}{*}{ Punição Inconsistente } & rho & 0,181 & 0,181 & $0,533 *$ & 0,334 \\
\hline & $\mathrm{p}$ & 0,503 & 0,503 & 0,033 & 0,207 \\
\hline \multirow{2}{*}{ Negligência } & rho & $-0,039$ & $-0,039$ & $-0,139$ & 0,15 \\
\hline & $\mathrm{p}$ & 0,887 & 0,887 & 0,608 & 0,58 \\
\hline \multirow{2}{*}{ Disciplina Relaxada } & rho & $-0,272$ & $-0,272$ & 0,025 & 0,081 \\
\hline & $\mathrm{p}$ & 0,309 & 0,309 & 0,926 & 0,765 \\
\hline \multirow{2}{*}{ Monitoria Negativa } & rho & $-0,064$ & $-0,064$ & 0,42 & 0,199 \\
\hline & $\mathrm{p}$ & 0,814 & 0,814 & 0,106 & 0,461 \\
\hline \multirow{2}{*}{ Abuso Físico } & rho & $-0,065$ & $-0,065$ & 0,071 & 0,131 \\
\hline & $\mathrm{p}$ & 0,81 & 0,81 & 0,793 & 0,629 \\
\hline
\end{tabular}

$* \mathrm{p} \leq 0,05 ; * * \mathrm{p} \leq 0,01$.

\section{Discussão}

É possível aferir que a maioria das correlações estabelecidas entre o IEP e os instrumentos de avaliação das funções executivas foram de magnitude baixa a moderada, grande parte das correlações encontradas se correlacionou com o Teste de Stroop Semântico. Duas hipóteses explicativas podem ser enumeradas, entre elas, tal ocorrido pode ser em função da amostra de sujeitos, pois devido à idade, todos os sujeitos foram avaliados com o Teste de Stroop Semântico e, apenas, metade da amostra foi avaliada com o Teste de Atenção por Cancelamento e Teste de Trilhas. Outro dado importante está relacionado com o fato das funções executivas serem multifatoriais, provavelmente o construto avaliado pelo IEP se correlacione mais com inibição, avaliada pelo Teste de Stroop Semântico. Segundo Miyake et al. (2000) e Diamond (2013), as funções executivas contemplam um construto composto por várias habilidades ou componentes, assim sendo, esperava-se grande número de correlações significativas, de baixas a moderadas, entre tais variáveis.

A partir do resultado de correlação negativa significativa entre o percentual do IEP e tempo de interferência no Teste de Stroop Semântico, pode-se observar que, quanto mais apropriado o estilo parental proporcionado pelos pais (medido como percentual no IEP), melhor o controle de interferência por parte dos filhos (menor a diferença no tempo de reação na parte incongruente do Teste de Stroop Semântico em relação à parte congruente). Para compreender, de forma aprofundada, a natureza dessa relação, é relevante observar os resultados das correlações entre funções executivas e as práticas educativas do IEP.

Das sete práticas educativas, apenas duas apresentaram alguma correlação significativa com as funções executivas, a saber, punição inconsistente e abuso físico. Em relação à punição inconsistente, ou seja, quando os pais se orientam por seu humor na hora de punir ou reforçar e não pelo ato praticado (Gomide, 2011b), houve correlação com as funções executivas. Observouse que, quanto maior a inconsistência na punição, mais a criança demora na parte incongruente do Teste de Stroop Semântico (tempo de reação na parte incongruente), mais ela é afetada pela incongruência (maior o tempo de reação de interferência no Teste de Stroop Semântico), porém maior é a sua flexibilidade (escore na parte $\mathrm{B}$ do Teste de Trilhas). Dentro desta prática educativa, há itens como os de número 17 (quando os pais estão nervosos castigam os filhos e quando passa pedem desculpas), 24 (quando estão nervosos descontam nos filhos) e 38 (quando estão mal humorados não deixam os filhos brincarem com os amigos). 
Outra prática educativa que merece destaque é a nomeada de abuso físico, caracterizada pela disciplina através de práticas corporais negativas, ameaça e chantagem de abandono e de humilhação do filho (Gomide, 2011b). Um item que exemplifica essa prática é o item 14, que contempla se o filho tem medo de apanhar dos pais. Essa prática educativa também se correlacionou com o desempenho no Teste de Stroop Semântico: quanto maior o uso de abuso físico, mais a criança demora na parte incongruente do Stroop (tempo de reação na parte incongruente) e mais ela é afetada pela incongruência (maior o tempo de reação de interferência no Teste de Stroop Semântico).

Por fim, conforme informações detalhadas em relação ao IEP, a prática educativa nomeada de punição inconsistente e abuso físico, apresentaram maior número de correlações significantes. Alguns estudos confirmam tais dados elencados, como, por exemplo, a pesquisa de Bernier et al. (2012), que relata sobre as estratégias ensinadas pelo cuidador de forma apropriada, as quais podem ser relacionadas à distração com uma atividade agradável enquanto se espera por algo ou ao resolver um quebra-cabeça voltar atrás e considerar outras opções para resolvê-lo, resultam em interações familiares com um clima emocional harmonioso.

Assim, repetidas experiências de regulação de sucesso em determinadas situações emocionalmente desgastantes, por parte dos progenitores, levam as crianças a internalizar as competências adquiridas e gradualmente integrá-las em seu próprio repertório de autorregulação. Desta forma, os processos regulatórios são, primeiramente, praticados no âmbito das relações familiares e as estratégias aprendidas são generalizadas e utilizadas fora da relação didática, tal como durante tarefas que exijam autorregulação independente, a qual pode ser considerada uma característica definidora das tarefas de funções executivas (Bernier et al., 2012). Pode-se supor, por meio do estudo de Bernier et al. (2012), que os fatores mais proximais, como por exemplo, as funções executivas dos próprios pais, possam afetar o controle das crianças referente ao próprio impulso, o qual pode ser por meio de imitação e de aprendizagem por observação.

Também a pesquisa de Rhoades et al. (2011) encontrou achados semelhantes, revelando que os riscos familiares advindos de questões demográficas podem prever as funções executivas em crianças. Os autores abordaram o tema abuso físico como uma prática inclusa no denominado "perfil familiar de risco" e revelaram que combinações específicas de risco são mais associadas com baixos resultados de funções executivas e que as mesmas podem variar de acordo com a raça, estado civil e condição socioeconômica. Para estes autores, pode-se esperar que esse estilo parental seja mais intrusivo devido aos seus níveis mais elevados de controle parental e, geralmente, estão associados a um baixo desempenho em relação ao desenvolvimento infantil. Observou-se, por exemplo, que a qualidade da parentalidade pode exercer influência no desenvolvimento das crianças de duas maneiras. Em primeiro lugar, por meio de interações sensíveis contingentes com os progenitores, afinal, por meio destas, a criança pode aumentar a sua motivação para aprender a controlar e interagir com o mundo externo, o que pode contribuir para a prática das habilidades relacionadas às funções executivas. Em um segundo momento, por meio da segurança derivada de interações positivas, neste caso as crianças são mais propensas a explorar o seu ambiente, aumentando a sua interação com materiais que a estimulem cognitivamente (Rhoades et al., 2011). Dessa forma, o contexto social pode promover a exposição a interações cognitivamente estimulantes que, por sua vez, podem promover as funções executivas.

Apesar de tais achados previamente relatados estarem em consonância com o esperado teoricamente, observou-se resultado inesperado em relação à flexibilidade cognitiva, que tendeu a ser maior quando há punição inconsistente. Contrariamente ao aqui observado, estudos prévios têm consistentemente relacionado maior caos doméstico e maior instabilidade familiar a menor funções executivas em crianças (Bernier et al., 2012; Hughes \& Ensor, 2009; Rhoades et al., 2011). Porém tais pesquisas não analisaram, especificamente, a flexibilidade cognitiva das crianças, o que dificulta a comparação com os presentes achados.

Hughes e Ensor (2009), por exemplo, chegaram à conclusão de que vários aspectos das interações sociais sustentam as diferenças individuais das funções executivas em crianças pequenas. Dentre várias características familiares analisadas, as que predisseram as funções executivas em crianças britânicas de 4 anos foram habilidade de planejamento da mãe, suporte (scaffolding) oferecido pela mãe e caos familiar, e a predição por estilo parental inconsistente foi marginalmente significativa na predição. No estudo, porém, foi usada uma única medida de funções executivas, derivada de quatro diferentes tarefas, não sendo possível proceder à análise diferencial da relação entre características familiares e cada aspecto das funções executivas.

Exploratoriamente, pode-se hipotetizar que a flexibilidade cognitiva pode estar relacionada com o fato das crianças que vivem sob a prática educativa de 
punição inconsistente não saberem reconhecer quais os comportamentos desejados pelos pais, apenas sabem que o humor dos mesmos varia (Gomide, 2011a). É possível que a flexibilidade seja desenvolvida em função da instabilidade do ambiente, sendo que as crianças devem estar sempre atentas para responderem aos comportamentos imprevisíveis dos seus progenitores. Porém, tal resultado não era esperado a partir do referencial teórico ou empírico da área e constituise em uma observação a ser analisada em estudos futuros, podendo ser apenas um viés da amostra aqui analisada.

\section{Considerações Finais}

O presente estudo buscou investigar a relação entre estilos parentais e as funções executivas em crianças de 3 a 6 anos de idade. Dentre as relações observadas, os resultados revelaram que, de modo geral, quanto mais apropriado o estilo parental dos pais, tanto melhor o controle de interferência dos filhos e que, quanto maior a inconsistência na punição, menor a capacidade de inibição da criança. Porém, um resultado inesperado foi observado, que quanto maior a inconsistência na punição, maior a sua flexibilidade cognitiva.

Algumas limitações devem ser consideradas neste estudo e podem, potencialmente, ser explicativas dos resultados observados, especialmente dos inesperados. Entre elas, a principal é o tamanho amostral, que é restrito e limitador para a generalização dos resultados. Ressalta-se ainda que o status socioeconômico das famílias e o nível de estudo dos progenitores foi bastante homogêneo na amostra avaliada, o que pode dificultar a generalização dos resultados, ainda que se considerem que as famílias podem apresentar estruturas e dinâmicas diferentes, de modo que tal aspecto deve ser investigado em pesquisas futuras com o objetivo de minimizar possíveis vieses. Finalmente, ressalta-se que os instrumentos de avaliação das funções executivas também podem ser considerados como uma limitação ao pensar que nem todos abordam a faixa etária em questão. Sugere-se que futuramente sejam elaborados e validados instrumentos para uma faixa etária precoce do desenvolvimento infantil e com níveis socioeconômicos mais variados, além de novos estudos com análises de regressão em amostras maiores.

\section{Referências}

Ardila, A. (2008). On the evolutionary origins of executive functions. Brain and Cognition, 68(1), 92-99. http://dx.doi. org/10.1016/j.bandc.2008.03.003

Ardila, A., Rosselli, M., Matute, E., \& Guajardo, S. (2005). The influence of the parents educational level on the development of executive functions. Developmental Neuropsychology, Norwich, 28(1), 539-560. http://dx.doi. org/10.1207/s15326942dn2801_5

Baron, I. S. (2004). Neuropsychological evaluation of the child. New York: Oxford University Press.

Bernier, A., Carlson, S. M., Deschênes, M., \& Matte-Gagné, C. (2012). Social factors in the development of early executive functioning: a closer look at the caregiving environment. Developmental Science, 15(1), 12-24. http://dx.doi. org/10.1111/j.1467-7687.2011.01093.x

Bernier, A., Whipple, N., \& Carlson, S. M. (2010). From external regulation to self- regulation: early parenting precursors of young children's executive functioning. Child Development, 81(1), 326-339. http://dx.doi.org/10.1111/j.14678624.2009.01397.x

Berwid, O. G., Curko Kera, E. A., Marks, D. J., Santra, A., Bender, H. A., \& Halperin, J. M. (2005). Sustained attention and response inhibition in young children at risk for Attention Deficit/Hyperactivity Disorder. Journal of Child Psychology \& Psychiatry, 46(11), 1219-1229. http://dx.doi.org/10.1111/j.1469-7610.2005.00417.x

Best, J. R. \& Miller, P. H. (2010). A developmental perspective on executive function. Child Development, 81(6), 16411660. http://dx.doi.org/10.1111/j.1467-8624.2010.01499.x

Bibok, M. B., Carpendale, J. I. M., \& Müller, U. (2009). Parental scaffolding and the development of executive function. In: Lewis, C. \& Carpendale, J. I. M. (Eds.). Social interaction and the development of executive function. New Directions in Child and Adolescent Development, 123. http://dx.doi.org/10.1002/cd.233

Bindman, S. W., Hindman, A. H., Bowles, R. P., \& Morrison, F. J. (2013). The contributions of parental management language to executive function in preschool children. Early Childhood Research Quarterly, 28(3), 529-539. http:// dx.doi.org/10.1016/j.ecresq.2013.03.003

Brocki, K. C. \& Bohlin, G. (2006). Developmental change in the relation between executive functions and symptoms of $\mathrm{ADHD}$ and co-occurring behavior problems. Infant and Child Development, 15(1), 19-40. http://dx.doi.org/10.1002/ icd. 413

Carvalho, J., Martins, C., Martins, E. C., Osório, A., Carvalho, M. J., \& Soares, I. (2012). Scaffolding verbal materno no âmbito de uma tarefa de elicitação narrativa em crianças de idade pré-escolar. Análise Psicológica, 30(4), $359-371$. http://dx.doi.org/10.14417/ap.598 
Czermainski, F. R., Bosa, C. A., \& Salles, J. F. (2013). Funções executivas em crianças e adolescentes com transtorno do espectro do autismo: uma revisão. Psico, 44(4), 518-525.

Darling, N. \& Steinberg, L. (1993). Parenting style as context: an integrative model. Psychological Bulletin, 113(3), 487-496. http://dx.doi.org/10.1037/0033-2909.113.3.487

Diamond, A. (2013). Executive functions. Annual Review of Psychology, 64, 135-168. http://dx.doi.org/10.1146/annurevpsych-113011-143750

Dias, N. M. \& Seabra, A. G. (2013). Funções executivas: desenvolvimento e intervenção. Temas sobre Desenvolvimento, 19(107), 206-212.

Dias, N. M., Seabra, A. G. (2015). The promotion of executive functioning in a Brazilian public school: a pilot study. Spanish Journal of Psychology, 18(e8), 1-14. http://dx.doi.org/10.1017/sjp.2015.4

Dias, N. M., Gomes, C. M. A., Reppold, C. T., Fioravanti-Bastos, A. C. M., Pires, E. U., Carreiro, L. R. R., \& Seabra, A. G. (2015). Investigação da estrutura e composição das funções executivas: análise de modelos teóricos. Revista Psicologia: Teoria e Prática, 17(2), 140-152. http://dx.doi.org/10.15348/1980-6906/psicologia.v17n2p140-152

Dias, N. M., Trevisan, B. T., Pereira, A. P. P., Gonzales, M. K., \& Seabra, A. G. (2012). Dados normativos do teste de atenção por cancelamento. In Seabra, A. G. \& Dias, N. M. (Org.), Avaliação Neuropsicológica Cognitiva: atenção e funções executivas. São Paulo: Memnon.

Espy, K, A. (1997). The Shape School: Assessing executive function in preschool children. Developmental Neuropsychology, 13(4), 495-499. http://dx.doi.org/10.1080/87565649709540690

Espy, K, A. \& Cwik, M. F. (2004). The Development of a trail making test in young children: the TRAILS-P. The Clinical Neuropsychologist, 18(3), 411-422. http://dx.doi.org/10.1080/138540409052416

Espy, K. A., Kaufmann, P. M. \& Glisky, M. L. (2001). New procedures to assess executive functions in preschool children. The Clinical Neuropsychologist, 15(1), 46-58. http://dx.doi.org/10.1076/clin.15.1.46.1908

Gerstadt, C. L., Hong, Y. J., \& Diamond, A. (1994). The relationship between cognition and action: performance of children 3.5-7 years old on a Stroop-like day-night test. Cognition, 53(2), 129-153. http://dx.doi.org/10.1016/00100277(94)90068-X

Godoy, S. (2012). Evidências de validade do teste de atenção por cancelamento. In Seabra, A. G. \& Dias, N. M. (Org.), Avaliação Neuropsicológica Cognitiva: atenção e funções executivas. São Paulo: Memnon.

Gomide, P. I. C. (2011a). Pais presentes pais ausentes: regras e limites. Rio de Janeiro: Vozes.

Gomide, P. I. C. (2011b). Inventário de Estilos Parentais - IEP: modelo teórico, manual de aplicação, apuração e inter-

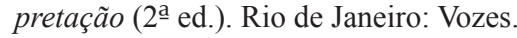

Hughes, C. H. \& Ensor, R. A. (2009). How do families help or hinder the emergence of early executive function? New Directions in Child and Adolescent Development, 123. http://dx.doi.org/10.1002/cd.234

Leme, V. B. R., Del Prette, Z. A. P., \& Coimbra, S. (2013). Práticas Educativas Parentais e Habilidades Sociais de Adolescentes de Diferentes Configurações Familiares. Psico, 44(4), 560-570.

Lezak, M. D., Howieson, D. B., Bigler, E. D., \& Tranel, D. (2012). Neuropsychological Assessment (5a ed.). New York: Oxford University Press.

Malloy-Diniz, L. F., Sedo, M., Fuentes, D., \& Leite, W. B. (2008). Neuropsicologia das funções executivas. In Fuentes, D., Malloy-Diniz, L. F., Camargo, C. H. P., \& Cosenza, R. M. (Org.), Neuropsicologia: teoria e prática. Porto Alegre: Artmed.

Martoni, A. T. (2012). Avaliação de funções executivas, desatenção e hiperatividade em crianças: testes de desempenho, relato de pais e de professores. Dissertação de mestrado, Universidade Presbiteriana Mackenzie, São Paulo, Brasil.

Miyake, A., Friedman, N. P., Emerson, M. J., Witzki, A. H., Howerter, A., \& Wager, T. D. (2000). The unity and diversity of executive functions and their contributions to complex "frontal lobe" tasks: a latente variable analysis. Cognitive Psychology, 41(1), 49-100. http://dx.doi.org/10.1006/cogp.1999.0734

Montiel, J. M. \& Seabra, A. G. (2012). Teste de atenção por cancelamento. In Seabra, A. G. \& Dias, N. M. (Org.), Avaliação Neuropsicológica Cognitiva: atenção e funções executivas. São Paulo: Memnon.

Montiel, J. M., Bartholomeu, D., Armond, G. D., Jacini, W. F. S., Bueno, C. H., Fernandes, F., \& Cecato, J. F. (2014). Associações entre medidas de funções executivas e sintomas de desatenção e hiperatividade em crianças em idade escolar. Revista Neuropsicologia Latinoamericana, 6(1), 13-21.

Noble, K., Mccandliss, B., \& Farah, M. (2007). Socioeconomic gradients predict individual differences in neurocognitive abilities. Developmental Science, 10(4), 464-480. http://dx.doi.org/10.1111/j.1467-7687.2007.00600.x

Osório, A. A. C. (2011). Development of social cognition in the early years of life in the context of the child-mother relationship. Tese de Doutorado em Psicologia Clínica, Universidade do Minho, Braga.

Oxford, M. L. \& Lee, J. O. (2011). The effect of family processes on school achievement as moderated by socioeconomic context. Journal of School Psychology, USA, 49(5), 597-612. http://dx.doi.org/10.1016/j.jsp.2011.06.001

Partington, J. E. \& Leiter, R. G. (1949). Partington's Pathways Test. Journal of Consulting Psychology, 13(5), 386. http:// dx.doi.org/10.1037/h0049525

Rhoades, B. L., Greenberg, M. T., Lanza, S. T., \& Blair, C. (2011). Demographic and familial predictors of early executive function development: contribution of a person-centered perspective. Journal of Experimental Child Psychology, 108(3), 638-662. http://dx.doi.org/10.1016/j.jecp.2010.08.004 
Sampaio, I. T. A. \& Gomide, P. I. C. (2007). Inventário de Estilos Parentais (IEP) - Gomide (2006) percurso de padronização e normatização. Psicologia Argumento, Curitiba, 25(48), 15-26.

Sarsour, K., Sheridan, M., Jutte, D., Nuru-Jeter, A., Hinshaw, S. \& Boyce, T. (2011). Family socioeconomic status and child executive functions: the roles of language, home environment, and single parenthood. Journal of the International Neuropsychological Society, 17, 120-132. http://dx.doi.org/10.1017/S1355617710001335

Stroop, J. R. (1935). Studies of interference in serial verbal reactions. Journal of Experimental Psychology, 18(6), 643-662. http://dx.doi.org/10.1037/0096-3445.121.1.15

Trevisan, B. T. (2010). Atenção e controle inibitório em pré-escolares e correlação com indicadores de desatenção e hiperatividade. Dissertação de mestrado, Universidade Presbiteriana Mackenzie, São Paulo, Brasil.

Trevisan, B. T., Hipólito, R., Parise, L. F., Reppold, C. T. \& Seabra, A. G. (2012). Dados normativos do teste de trilhas para pré-escolares. In Seabra, A. G. \& Dias, N. M. (Org.), Avaliação Neuropsicológica Cognitiva: atenção e funções executivas. São Paulo: Memnon.

Trevisan, B. T. \& Pereira, A. P. P. (2012). Evidências de validade do teste de trilhas para pré-escolares. In Seabra, A. G. \& Dias, N. M. (Org.), Avaliação Neuropsicológica Cognitiva: atenção e funções executivas. São Paulo: Memnon.

Trevisan, B. T. \& Seabra, A. G. (2012). Teste de trilhas para pré-escolares. In Seabra, A. G. \& Dias, N. M. (Org.), Avaliação Neuropsicológica Cognitiva: atenção e funções executivas. São Paulo: Memnon.

Autores:

Gabriela Lamarca Luxo Martins - Doutoranda, Universidade Presbiteriana Mackenzie

Camila Barbosa Riccardi León - Doutoranda, Universidade Presbiteriana Mackenzie.

Alessandra Gotuzo Seabra - Doutora, Universidade Presbiteriana Mackenzie.

Endereço para correspondência:

Gabriela Lamarca Luxo Martins

Rua Itapicuru, 699 apto. 103 - Perdizes

05006-000 São Paulo, SP, Brasil

Recebido em: 19.11 .15

Aceito em: 29.05.16 